\title{
Carbon Nanotube Radio
}

\author{
Ujjal Kumar Sur \\ Department of Chemistry, Behala College, \\ India
}

\section{Introduction}

Carbon nanotubes (CNTs) are allotropes of carbon with a cylindrical nanostructure. Nanotubes have been synthesized with length-to-diameter ratio of up to $132,000,000: 1$, which is significantly greater than any other material. These cylindrical carbon molecules have novel properties which make them potentially useful in many applications in nanotechnology, electronics, optics, and other fields of materials science.

Nanotubes are members of the fullerene structural family (one dimensional form of fullerenes), which also includes the spherical buckyballs. Fullerenes were accidentally discovered by Kroto and Smalley in 1985, when they found strange results in mass spectra of evaporated carbon samples (Kroto et al. 1985). Since the discovery in 1991 by Iijima (Iijima, 1991), carbon nanotubes have been investigated by many researchers all over the world both from fundamental point of view and for future potential applications in the field of energy storage, molecular electronics, nanomechanical devices and composite materials. However, the real applications are still under development. Their name is derived from their size, since the diameter of a nanotube is on the order of a few nanometers (approximately 1/50,000th of the width of a human hair), while they can be up to 18 centimeters in length. Nanotubes are categorized as single-walled nanotubes (SWNTs) and multi-walled nanotubes (MWNTs). Figures 1 a) and b) show the scanning tunneling microscopic (STM) and transmission electron microscopic (TEM) images of a single-walled carbon nanotube. Figure 2 illustrates the scanning electron microscopic (SEM) image of carbon nanotube bundles. Single-walled nanotubes are an important variety of carbon nanotube because they exhibit electric properties that are not shared by the multi-walled carbon nanotube (MWNT). In particular, their band gap can vary from zero to about $2 \mathrm{eV}$ and their electrical conductivity can show metallic or semiconducting behavior, whereas MWNTs are zero-gap metals. Single-walled nanotubes are the most likely candidate for miniaturizing electronics beyond the micro electromechanical scale currently used in electronics. The most basic building block of these systems is the electric wire, and SWNTs can be excellent conductors.

One useful application of SWNTs is in the development of the first intramolecular field effect transistors (FET). Multi-walled nanotubes (MWNTs) consist of multiple rolled layers (concentric tubes) of graphite. There are two models which can be used to describe the structures of multi-walled nanotubes. Single Walled Nanotubes (SWNTs) can be considered as long wrapped graphene sheets.

As stated before, nanotubes generally have a length to diameter ratio of about 1000 so they can be considered as nearly one-dimensional structures. Figure 3 shows some SWNTs with 
different chiralities. The difference in structure is shown at the open end of the tubes. More detailed, a SWNT consists of two separate regions with different physical and chemical properties. The first is the sidewall of the tube and the second is the end cap of the tube. The end cap structure is similar to or derived from a smaller fullerene, such as C60. Multi Walled Nanotubes (MWNTs) can be considered as a collection of concentric SWNTs with different diameters. The length and diameter of these structures differ a lot from those of SWNTs and, of course, their properties are also very different. Figure 4 illustrates the schematic diagrams of fullerenes, SWNTs and MWNTs. Electronic, molecular and structural properties of carbon nanotubes are determined to a large extent by their nearly one dimensional structure. The most important properties of CNTs and their molecular background are stated below.
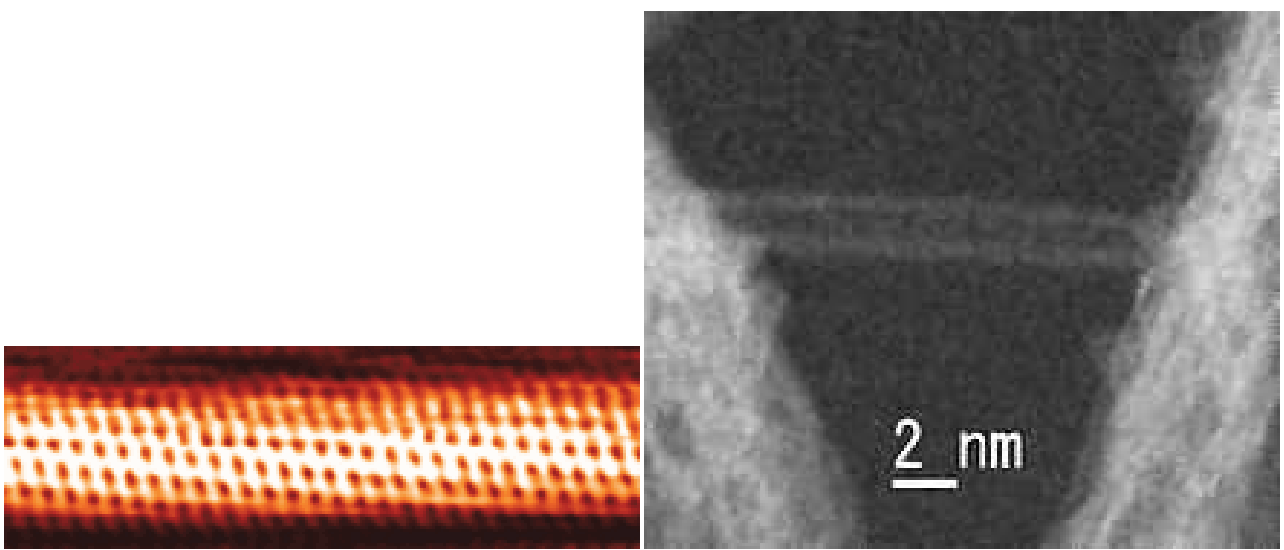

Fig. 1. a) An STM image of a single-walled carbon nanotube, b) Transmission electron microscopic image showing a single-walled carbon nanotube.

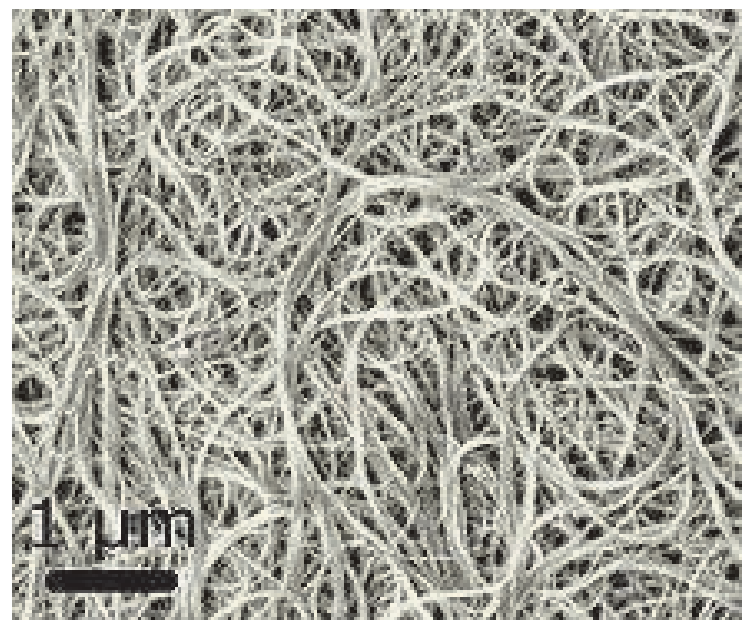

Fig. 2. Scanning electron microscopy image of carbon nanotube bundles. 

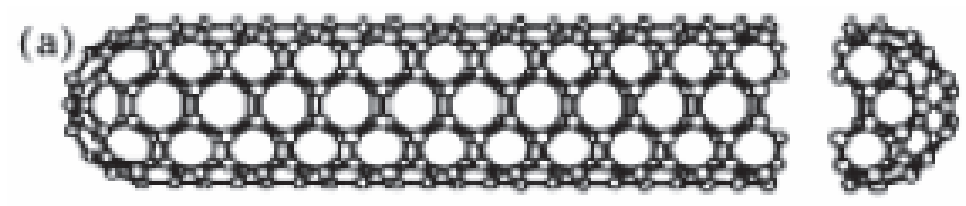

(b)
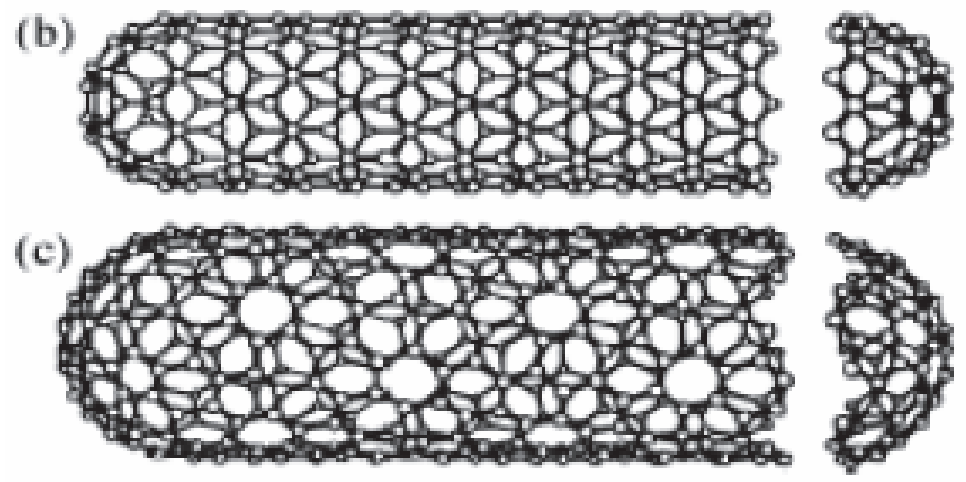

Fig. 3. Some SWNTs with different structures. (a) armchair structure (b) zigzag structure (c) chiral structure.

\section{Properties of carbon nanotube}

\subsection{Chemical reactivity}

The chemical reactivity of a CNT is, compared with a graphene sheet, enhanced as a direct result of the curvature of the CNT surface. Carbon nanotube reactivity is directly related to the pi-orbital mismatch caused by an increased curvature. Therefore, a distinction must be made between the sidewall and the end caps of a nanotube. For the same reason, a smaller nanotube diameter results in increased reactivity. Covalent chemical modification of either sidewalls or end caps has shown to be possible and the solubility of CNTs in different solvents can be controlled by this way. Though, direct investigation of chemical modifications on nanotube behaviour is difficult as the crude nanotube samples are still not pure enough.

\subsection{Electrical conductivity}

Depending on their chiral vector, carbon nanotubes with a small diameter are either semiconducting or metallic. The differences in conducting properties are caused by the molecular structure that results in a different band structure and thus a different band gap. The differences in conductivity can easily be derived from the graphene sheet properties.

\subsection{Optical activity}

Theoretical studies have shown that the optical activity of chiral nanotubes disappears when the nanotubes become larger.Therefore, it is expected that other physical properties are influenced by these parameters too. Use of the optical activity might result in optical devices in which CNTs play an important role. 


\subsection{Mechanical strength}

Carbon nanotubes have a very large Young modulus in their axial direction. The nanotube as a whole is very flexible because of the great length. Therefore, these compounds are potentially suitable for applications in composite materials that need anisotropic properties.

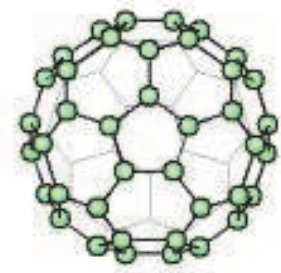

(a) $\mathrm{C}_{60}$

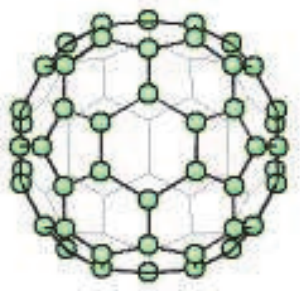

(b) $\mathrm{C}_{70}$

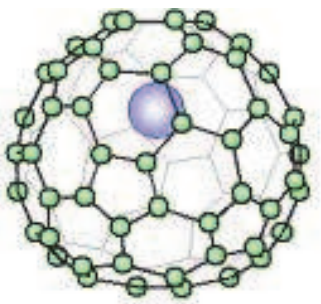

(c) $L a @ C_{\hat{\Delta} 2}$

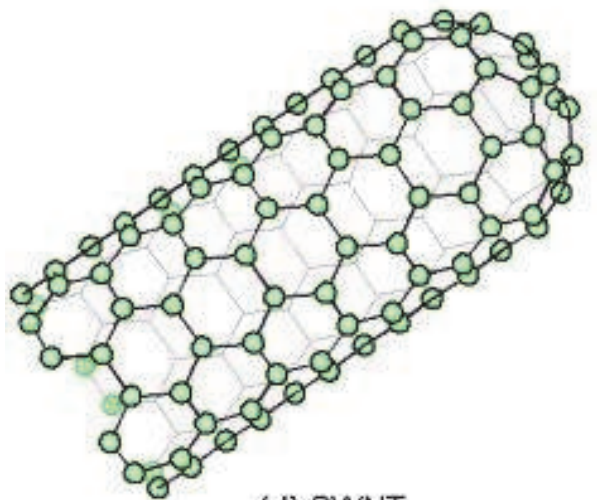

(d) SWNT

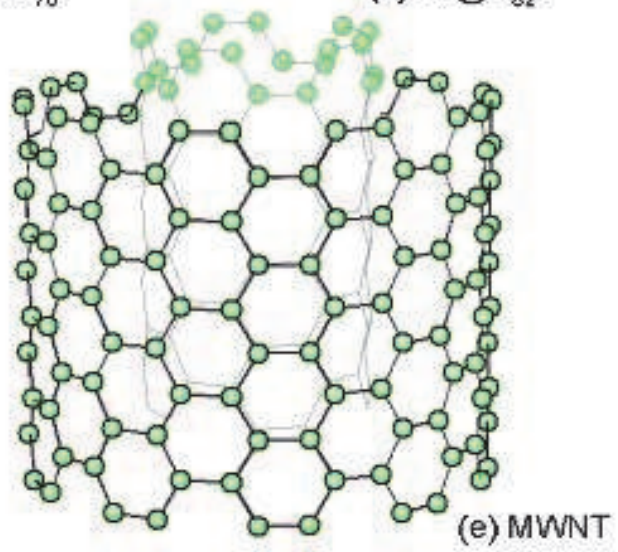

Fig. 4. Schematic diagrams of fullerenes and carbon nanotubes.

\section{Synthesis and purification of carbon nanotube}

\subsection{Synthesis}

Carbon nanotubes are generally synthesized by three main methods, arc discharge, laser ablation and chemical vapour deposition. In arc discharge technique, a vapour is created by an arc discharge between two carbon electrodes with or without catalyst. Nanotubes selfassemble from the resulting carbon vapour. In the laser ablation technique, a high-power laser beam impinges on a volume of carbon-containing feedstock gas (methane or carbon monoxide). At the moment, laser ablation produces a small amount of clean nanotubes, whereas arc discharge methods generally produce large quantities of impure material. In general, chemical vapour deposition (CVD) results in MWNTs or poor quality SWNTs. The SWNTs produced with CVD have a large diameter range, which can be poorly controlled. But on the other hand, this method is very easy to scale up, what favours commercial production. Table 1 shows a short summary of the three most common techniques for the synthesis of carbon nanotubes.

Figure 5 shows the photograph of the powder of carbon nanotube, while Figure 6 illustrates the growth of carbon nanotubes by plasma enhanced chemical vapour deposition method. 
Techniques have been developed to produce nanotubes in sizeable quantities, including arc discharge, laser ablation, high pressure carbon monoxide, and chemical vapor deposition (CVD). Most of these processes take place in vacuum or with process gases. CVD growth of CNTs can occur in vacuum or at atmospheric pressure. Large quantities of nanotubes can be synthesized by these methods; advances in catalysis and continuous growth processes are making CNTs more commercially viable

\begin{tabular}{|c|c|c|}
\hline Arc Discharge Method & Chemical Vapour Deposition & Laser Ablation \\
\hline $\begin{array}{l}\text { Introduced by Ajayan and } \\
\text { Ebbesen in } 1992\end{array}$ & Developed by Endo et al. in 1993 & $\begin{array}{ll}\text { Developed } & \text { by } \\
\text { Smalley in } 1995 & \end{array}$ \\
\hline Typical yield 30 to $90 \%$ & Typical yield 20 to $100 \%$ & $\begin{array}{l}\text { Typical yield upto } 70 \\
\%\end{array}$ \\
\hline $\begin{array}{l}\text { The method can easily } \\
\text { produce both SWNTs and } \\
\text { MWNTs }\end{array}$ & $\begin{array}{l}\text { Easiest method to produce large- } \\
\text { scale SWNTs }\end{array}$ & $\begin{array}{l}\text { This method can } \\
\text { produce SWNTs } \\
\text { with good diameter } \\
\text { control }\end{array}$ \\
\hline $\begin{array}{l}\text { This method is less } \\
\text { expensive }\end{array}$ & This method is less expensive & $\begin{array}{l}\text { This method is very } \\
\text { expensive }\end{array}$ \\
\hline $\begin{array}{l}\text { The reaction product is not } \\
\text { very pure and it requires } \\
\text { further purification }\end{array}$ & The reaction product is quite pure & $\begin{array}{l}\text { The reaction product } \\
\text { is quite pure }\end{array}$ \\
\hline
\end{tabular}

Table 1. A summary of the three common techniques for the synthesis of carbon nanotubes

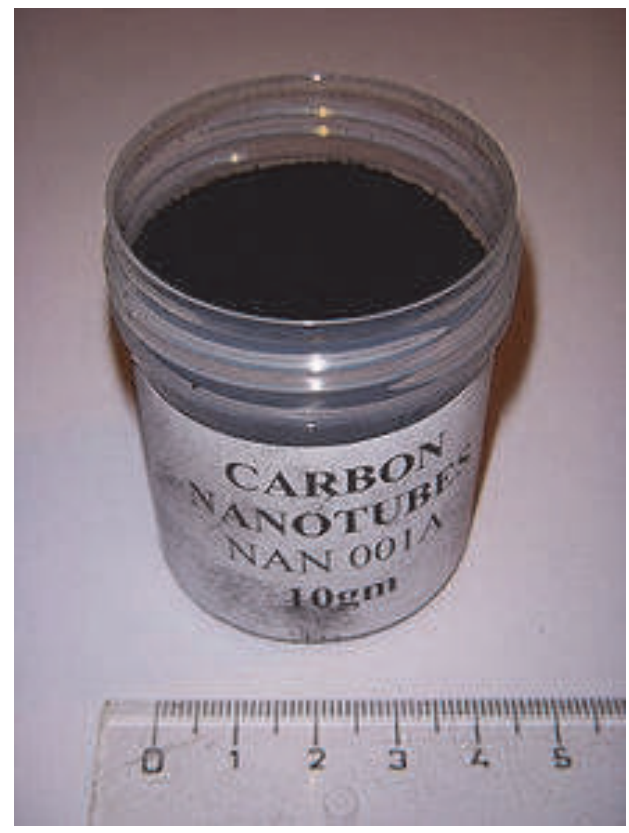

Fig. 5. Photograph of carbon nanotube powder 


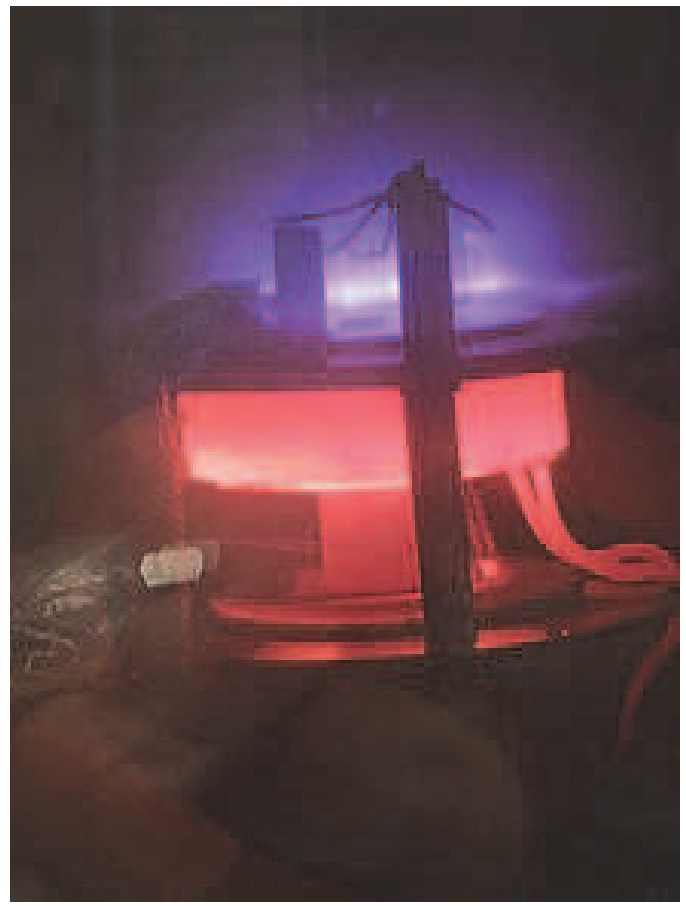

Fig. 6. Carbon nanotubes grown by plasma assisted CVD method

\subsubsection{Arc discharge method}

Nanotubes were observed in 1991 in the carbon soot of graphite electrodes during an arc discharge, by using a current of 100 amps, that was intended to produce fullerenes. However the first macroscopic production of carbon nanotubes was made in 1992 by Ajayan and Ebbesen (Ebbesen \& Ajayan, 1992). Because nanotubes were initially discovered using this technique, it has been the most widely-used method of nanotube synthesis. The yield for this method is up to 30 percent by weight and it produces both single- and multi-walled nanotubes with lengths of up to 50 micrometers with few structural defects.

\subsubsection{CVD method}

During CVD, a substrate is prepared with a layer of metal catalyst particles, most commonly nickel, cobalt, iron, or a combination of all. The metal nanoparticles can also be produced by other ways, including reduction of oxides or oxides solid solutions. The diameters of the nanotubes that are to be grown are related to the size of the metal particles. This can be controlled by patterned (or masked) deposition of the metal, annealing, or by plasma etching of a metal layer. The substrate is heated to approximately $700^{\circ} \mathrm{C}$. To initiate the growth of nanotubes, two gases are bled into the reactor: a process gas (such as ammonia, nitrogen or hydrogen) and a carbon-containing gas (such as acetylene, ethylene, ethanol or methane). Nanotubes grow at the sites of the metal catalyst; the carbon-containing gas is broken apart at the surface of the catalyst particle, and the carbon is transported to the edges of the particle, where it forms the nanotubes. 


\subsubsection{Laser ablation method}

In the laser ablation method, a pulsed laser vaporizes a graphite target in a hightemperature reactor while an inert gas is bled into the chamber. Nanotubes develop on the cooler surfaces of the reactor as the vaporized carbon condenses. A water-cooled surface may be included in the system to collect the nanotubes. This process was developed by Dr. Richard Smalley and co-workers at Rice University (Smalley et al. 1995). They used a composite of graphite and metal catalyst particles (the best yield was from a cobalt and nickel mixture) to synthesize single-walled carbon nanotubes. The laser ablation method yields around $70 \%$ and produces primarily single-walled carbon nanotubes with a controllable diameter determined by the reaction temperature. However, it is more expensive than either arc discharge or chemical vapor deposition

\subsection{Purification}

A major problem with nanotube application is the purification of the reaction product along with the large-scale synthesis. The as-produced SWNT soot contains a lot of impurities. The main impurities in the soot are graphite (wrapped up) sheets, amorphous carbon, metal catalyst and the smaller fullerenes. These impurities will interfere with most of the desired properties of the SWNTs. Also in the fundamental research, it is preferred to obtain SWNTs or the impurities, as pure as possible without changing them. In order to understand the measurements better, the SWNT samples also have to be as homogeneous as possible. The common industrial techniques use strong oxidation and acid refluxing techniques, which have an effect on the structure of the tubes.

\section{Potential applications of carbon nanotube}

\subsection{Energy storage}

Graphite, carbonaceous materials and carbon fibre electrodes are commonly used in fuel cells, batteries and other electrochemical applications. Advantages of considering nanotubes for energy storage are their small dimensions, smooth surface topology and perfect surface specificity. The efficiency of fuel cells is determined by the electron transfer rate at the carbon electrodes, which is the fastest on nanotubes following ideal Nernstian behaviour.

\subsubsection{Hydrogen storage}

The advantage of hydrogen as energy source is that its combustion product is water. In addition, hydrogen can be easily regenerated. For this reason, a suitable hydrogen storage system is necessary, satisfying a combination of both volume and weight limitations. The two commonly used means to store hydrogen are gas phase and electrochemical adsorption. Because of their cylindrical and hollow geometry, and nanometre-scale diameters, it has been predicted that carbon nanotubes can store a liquid or a gas in the inner cores through a capillary effect. It is reported that SWNTs were able to meet and sometimes exceed this level by using gas phase adsorption (physisorption). Yet, most experimental reports of high storage capacities are rather controversial so that it is difficult to assess the applications potential. Another possibility for hydrogen storage is electrochemical storage. In this case, not a hydrogen molecule but an $\mathrm{H}$ atom is adsorbed. This is called chemisorption. 


\subsubsection{Lithium intercalation}

The basic principle of rechargeable lithium batteries is electrochemical intercalation and deintercalation of lithium in both electrodes. An ideal battery has a high-energy capacity, fast charging time and a long cycle time. The capacity is determined by the lithium saturation concentration of the electrode materials. For Li, this is the highest in nanotubes if all the interstitial sites (inter-shell van der Waals spaces, inter-tube channels and inner cores) are accessible for Li intercalation. SWNTs have shown to possess both highly reversible and irreversible capacities. Because of the large observed voltage hysteresis, Li-intercalation in nanotubes is still unsuitable for battery application. This feature can potentially be reduced or eliminated by processing, i.e. cutting, the nanotubes to short segments. Carbon nanotubes are interesting intercalation hosts because of their structure and chemical bonding. Nanotubes might have a higher saturation composition than graphite as guest species can intercalate in the interstitial sites and between the nanotubes. Therefore, carbon nanotubes are expected to be suitable high energy density anode materials for rechargeable Li-ion batteries. Figure 7 shows the schematic diagram of graphite intercalated with lithium.

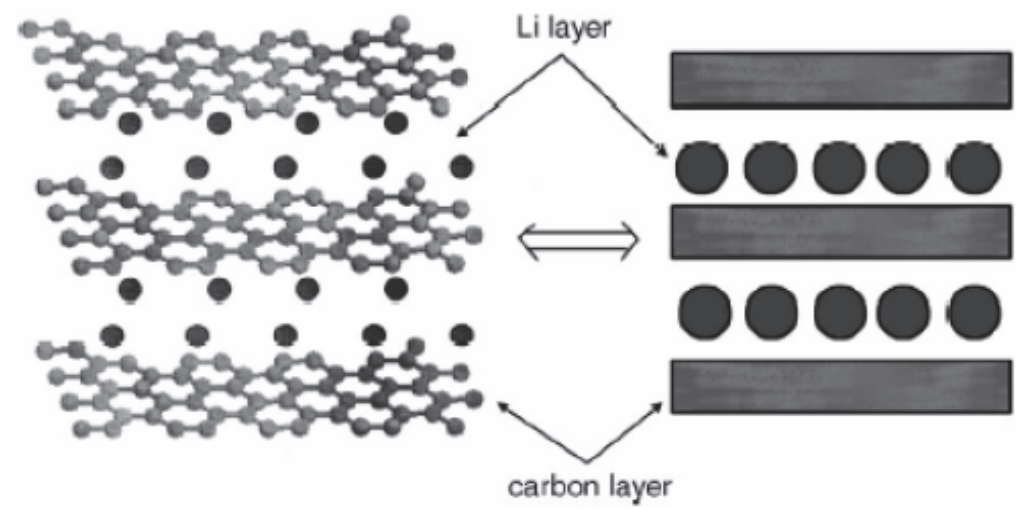

Fig. 7. Schematic diagram showing the intercalation of graphite with lithium

\subsubsection{Electrochemical supercapacitors}

Supercapacitors have a high capacitance and potentially applicable in electronic devices. Typically, they are comprised two electrodes separated by an insulating material that is ionically conducting in electrochemical devices. The capacity of an electrochemical supercapacitors inversely depends on the separation between the charge on the electrode and the counter charge in the electrolyte. Because this separation is about a nanometre for nanotubes in electrodes, very large capacities result from the high nanotube surface area accessible to the electrolyte. In this way, a large amount of charge injection occurs if only a small voltage is applied. This charge injection is used for energy storage in nanotube supercapacitors. Generally speaking, there is most interest in the double-layer supercapacitors and redox supercapacitors with different charge-storage modes. Carbon in general, and especially nanotubes, form an attractive material for electrochemical applications as they have a large active surface area. In addition, carbon is a relatively cheap, low density, environmentally friendly and highly polarisable material which makes application even more attractive. 
Carbon nanotubes have been proposed as electrodes for supercapacitors. Different values of capacitance mainly depend on the kind and purity of the samples. For purified nanotubes specific capacitance varies from 5 to $80 \mathrm{~F} / \mathrm{g}$. Pure carbon nanotubes have a moderate surface area (120 to $400 \mathrm{~m}^{2} / \mathrm{g}$ ) because of their highly mesoporous character. The more graphitised nanotubes show smaller values of capacitance. However, presence of defects causes an increase of ability for accumulation of charges. There is a great difference in capacitance between SWNTs and MWNTs. However, the many different ways of producing the nanotubes also create differences in capacitance values. To increase the capacitance of nanotubes it is possible to increase the electrode surface area or to increase the pseudocapacitance effects obtained by addition of special oxides or electrically conducting polymers (ECP) like polypyrrole (PPy). The ECPs have the advantage of lower costs compared to oxides. Another advantage is that the pseudocapacitance effects of ECPs are quite stable.

\subsection{Molecular electronics with CNTs}

\subsubsection{Field emitting devices}

If a solid is subjected to a sufficiently high electric field, electrons near the Fermi level can be extracted from the solid by tunneling through the surface potential barrier. This emission current depends on the strength of the local electric field at the emission surface and its work function (which denotes the energy necessary to extract an electron from its highest bounded state into the vacuum level). The applied electric field must be very high in order to extract an electron. This condition is fulfilled for carbon nanotubes, because their elongated shape ensures a very large field amplification. For technological applications, the emissive material should have a low threshold emission field and large stability at high current density. Furthermore, an ideal emitter is required to have a nanometer size diameter, a structural integrity, a high electrical conductivity, a small energy spread and a large chemical stability. Carbon nanotubes possess all these properties. However, a bottleneck in the use of nanotubes for applications is the dependence of the conductivity and emission stability of the nanotubes on the fabrication process and synthesis conditions. Examples of potential applications for nanotubes as field emitting devices are flat panel displays, gas discharge tubes in telecom networks, electron guns for electron microscopes, AFM tips and microwave amplifiers.

\subsubsection{Transistors}

The field-effect transistor-a three-terminal switching device-can be fabricated using only one semiconducting SWNT. By applying a voltage to a gate electrode, the nanotube can be switched from conducting to an insulating state. Such carbon nanotube transistors can be coupled together, working as a logical switch, which is the basic component of computers.

\subsubsection{Nanoprobes and sensors}

Because of their flexibility, nanotubes can also be used in scanning probe instruments. Since MWNT tips are conducting, they can be used in STM and AFM instruments. Advantages are the improved resolution in comparison with conventional Si or metal tips and the tips do not suffer from crashes with the surfaces because of their high elasticity. However, nanotube vibration, due to their large length, will remain an important issue until shorter nanotubes can be grown controllably. Nanotube tips can be modified 
chemically by attachment of functional groups. Because of this, nanotubes can be used as molecular probes, with potential applications in chemistry and biology. Other applications are the following:

- A pair of nanotubes can be used as tweezers to move nanoscale structures on surfaces.

- Sheets of SWNTs can be used as electromechanical actuators, mimicking the actuator mechanism present in natural muscles.

- $\quad$ SWNTs may be used as miniaturised chemical sensors.

\section{Carbon nanotube radio}

\subsection{Concept of radio device and historical background}

The radio is a device based on wireless transmission of signals by modulating the frequencies of radio waves. Since its invention, the radio has been playing an important role in modern civilization due to its use in the mass media (audio/video), radar, cellular phones and wireless computer network. Historically, the Indian physicist Sir Jagadish Chandra Bose started his pioneering research work on the generation/detection of millimetre wave radiation more than 100 years ago (Bose, 1897). In 1897, he demonstrated his apparatus to detect radio frequency (RF) in the Royal Institution, London. Some concepts from his original 1897 paper have been incorporated into the $1.3 \mathrm{~mm}$ receiver of the NRAO $12 \mathrm{~m}$ radio telescope at Tuscon, Arizona, USA.

James Clerk Maxwell was the first to mathematically conceive the idea of electromagnetic waves (in 1873). After him, in the late 1880s, Heinrich Hertz tried to produce electromagnetic waves which had optical properties, based on Maxwell's hypothesis that visible light is just a fraction of the total electromagnetic spectrum. Hertz, and later Popoff and Oliver Lodge were all using $66 \mathrm{~cm}$ waves. Bose, in the 1890s, revolutionised this field of research by producing $5 \mathrm{~mm}$ waves, called microwaves or millimetre waves. These could be polarized using smaller crystals, than would be required if $66 \mathrm{~cm}$ waves were used, and thus Bose could show that electromagnetic waves do indeed have optical properties. In 1895, Marconi built a wireless system capable of transmitting signals at long distances $(1.5 \mathrm{mi} . / 2.4 \mathrm{~km})$. In radio transmission technology, early public experimenters had made short distance broadcasts. Marconi achieved long range signalling due to a wireless transmitting apparatus and a radio receiver claimed by him. From Marconi's experiments, the phenomenon that transmission range is proportional to the square of antenna height is known as "Marconi's law". This formula represents a physical law that radio devices use. Marconi's experimental apparatus proved to be a complete, commercially successful radio transmission system. Shortly after the 1900s, Marconi held the patent rights for radio.

There was a gradual transformation in the dimension of radio from a bulky device with vacuum tube technology to a much smaller and sophisticated device based on solid state transistor technology. However, the transistor using silicon-based technology is reaching its physical limits. Carbon nanotube is an interesting nanomaterial with unique electrical and mechanical properties. Carbon nanotubes have been used as components in high-frequency electronics like high frequency field-effect transistors and RF detectors/mixers. Figure 8 shows the picture of the first wireless radio device fabricated by Professor Jagadish Chandra Bose (Figure 9 illustrates the image of Professor Bose). 


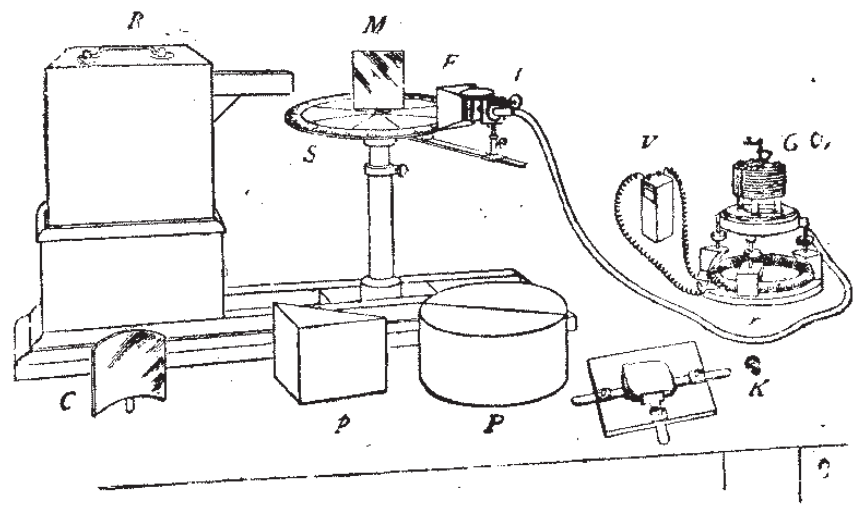

$R$, radiator ; $S$, spectrometer-circle : $M$, plane mirror ; $C$, cylindrical mirror ; $p$, totally reflecting prism; $P$, semi-cylinders; $\mathbb{K}$, crystal-holder; $F$, collecting funnel attached to the spiral spring receiver; $t$, tangent screw, by which the receiver is rotated; $V$, voltaic cell ; $r$, circular rheostat; $G$, galvanometer.

Fig. 8. Picture of the first radio device (measurement of millimeter waves) fabricated by Jagadish Chandra Bose

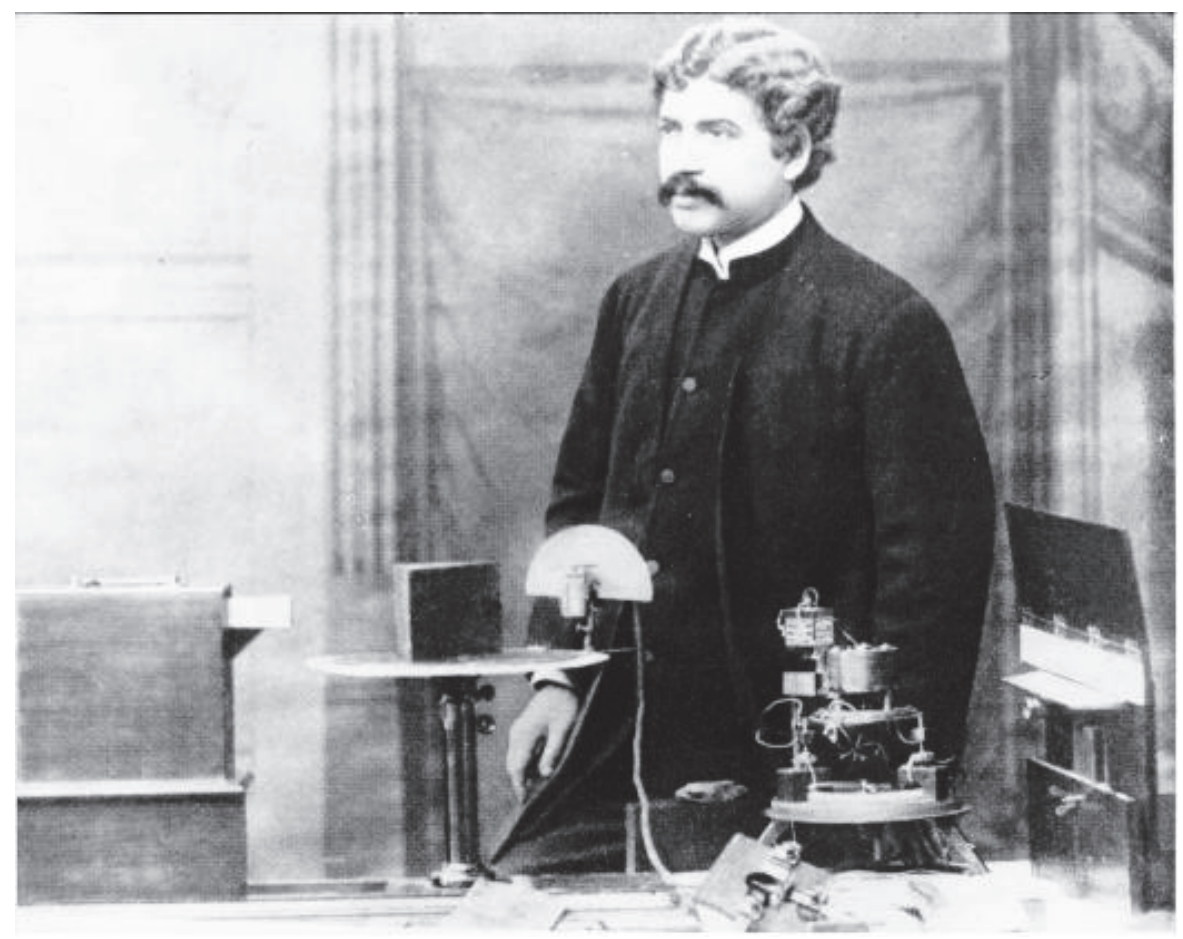

Fig. 9. Image of Professor Jagadish Chandra Bose 


\subsection{Development of carbon nanotube radio}

Researchers in California have reported the development of the world's first working radio device based on nanosized radio-wave receivers and detectors fabricated from a single carbon nanotube. Although, other researchers have fabricated nano-sized radio detectors in the past, the above-mentioned study illustrates the demonstration of the RF components in an actual working radio system for the first time. Zettl and coworkers from Lawrence Berkeley National Laboratory, University of California, Berkeley, USA, have fabricated a fully integrated radio receiver from a single carbon nanotube (Zettl et al. 2007). This radio receiver has dimension orders of magnitude lower than any previous radio. A single carbon nanotube served as all the four essential components (antenna, tuner, amplifier and demodulator) of a radio at the same time. In the nanotube radio, antenna and tuner can operate in a completely different way compared to the traditional radio. The antenna receives signals via high-frequency mechanical vibrations of the nanotube rather than electrically. These vibrations become significant only when the frequency of the incoming wave matches the resonance frequency of the nanotube. The resonance frequency of the nanotube can be tuned to receive only a preselected band of the electromagnetic spectrum. Field emission of electrons from the tip of the charged nanotube has been utilized to detect vibrations and to amplify/demodulate the signal. Simulation studies have shown the distribution of electric field surrounding the nanotube radio during the operation of the radio. The field is strongest at the tip of the nanotube and varies as the nanotube vibrates. This effect allows the nanotube to demodulate the radio signal.

Zettl and co-workers have demonstrated the coarse tuning of the nanotube radio from a low-frequency, frequency-modulated $(\mathrm{FM})$ radio band $(\sim 100 \mathrm{MHz})$, to much higher frequency bands $(\sim 350 \mathrm{MHz})$ along with the fully reversible fine tuning of resonance frequency of the nanotube. Carbon nanotubes with resonance frequencies from 10 to 400 $\mathrm{MHz}$ and quality factor $(Q) \sim 500$ were used in their experiments.

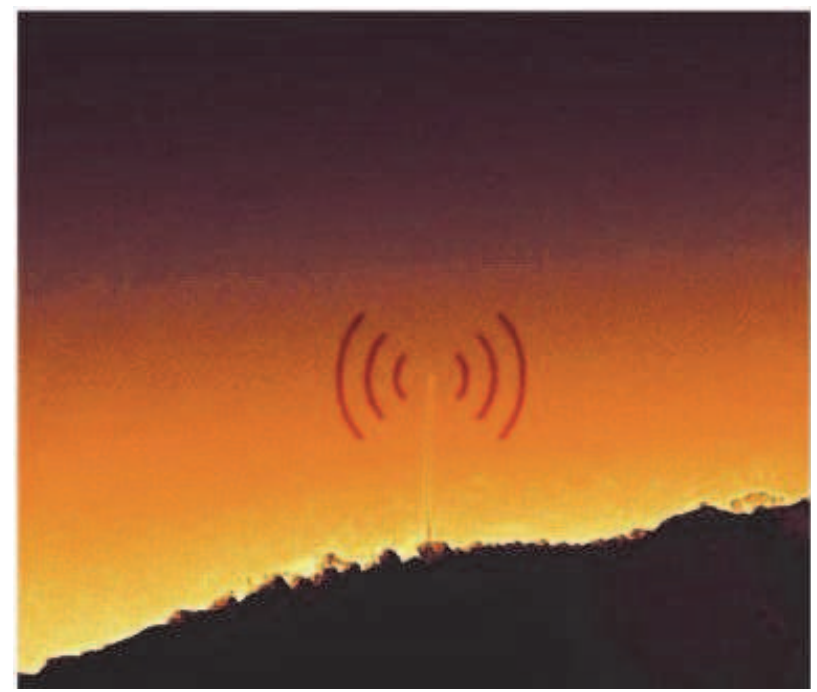

Fig. 10. TEM image of a single carbon nanotube protruding from an electrode. (Courtesy: Zettl and co-workers, Lawrence Berkeley National Laboratory and UC Berkeley, USA.) 
A high-resolution transmission electron microscope (TEM) allows them to observe the nanotube radio in action. The TEM image (Figure 10) shows a single carbon nanotube of 1 $\mu \mathrm{m}$ length and $10 \mathrm{~nm}$ width protruding from an electrode. A direct current voltage source (battery) has been connected to the electrodes as a power source for the operation of the radio in vacuum of the order of 10-7 Torr. The nanotube starts to vibrate vigorously when a radio wave of specific frequency impinges on it. The nanotube radio has been used to receive and play music from FM radio transmissions and it faithfully reproduced the audio signal and the music was clearly audible to the human ear. Figure 11 illustrates the gradual transformation in size of the radio with time. It began with the Philco vacuum tube radio of the 1930s to the pocket-sized transistor radio of the 1950s and the recent single-chip radio found in cellphones and wireless sensors. This single nanotube radio is about 19 orders of magnitude smaller than the Philco vacuum tube radio of the 1930s.

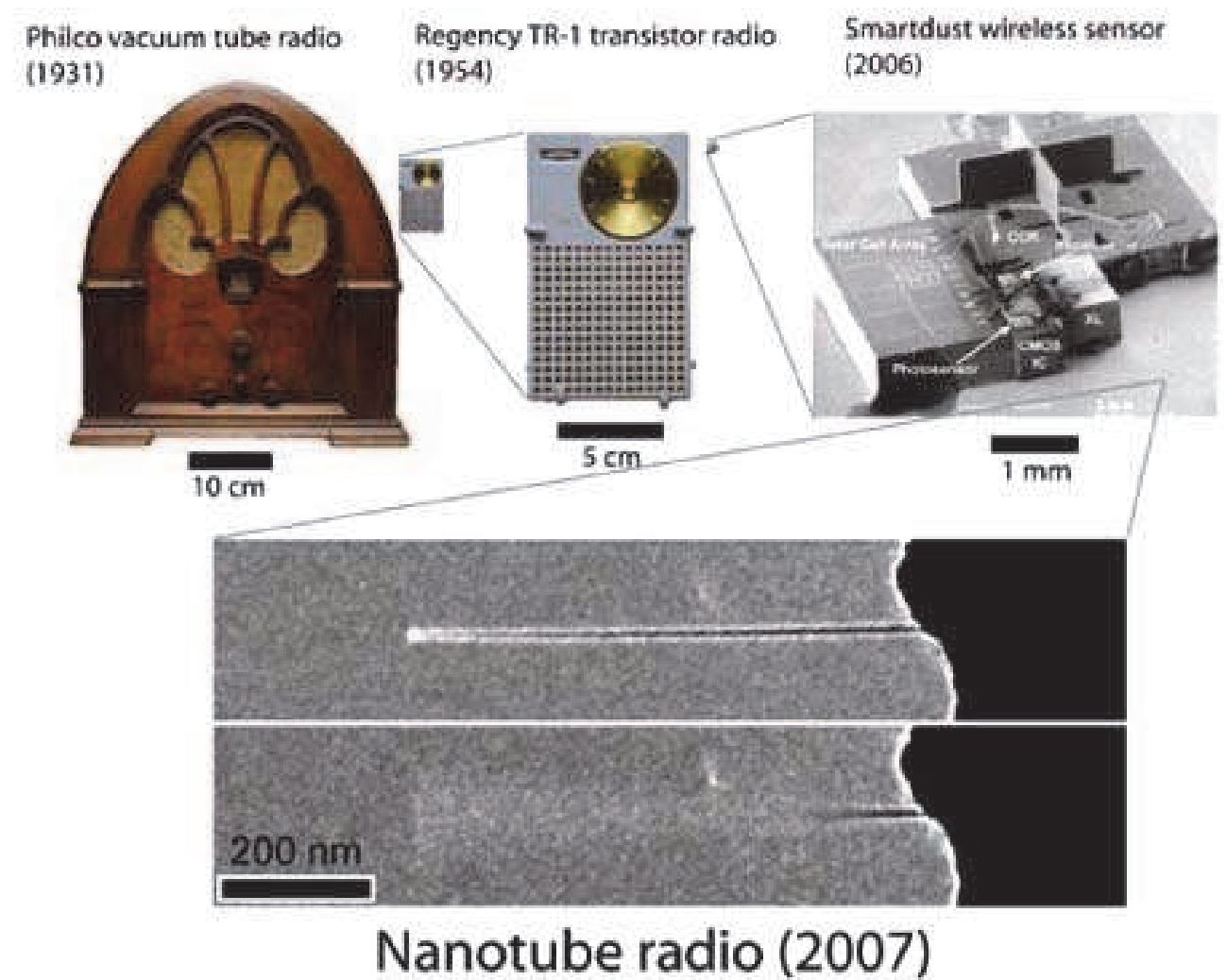

Fig. 11. Gradual miniaturization of radio with time. (Courtesy: Zettl and co-workers,Lawrence Berkeley National Laboratory and UC Berkeley, USA.)

Rutherglen and Burke from the University of California, Irvine, USA, have demonstrated a carbon nanotube to demodulate amplitude modulated signal with frequencies up to 100 $\mathrm{kHz}$ (Rutherglen \& Burke, 2007). Their device represents a room-temperature, two-terminal nonlinear device, simpler than the previous three-terminal mixers/detectors. They 
incorporated the detector into a complete radio system for successful wireless transmission of music from an iPod to a speaker several feet away from the music player. The audio quality of the signal demodulated by the carbon nanotube was clear to the human ear. Their study demonstrates the possibility of fabricating other components of the radio at nanometer scale in the near future. This may finally lead to a fully integrated nanoscale wireless communication system.

Due to the small dimension, such a nanoscale device has potential medical applications. The nanotube radio can be inserted inside the living human cell to give a subcellular remote controlled interface. This special kind of nanotube radio can be utilized to image inside the cells in real time and under normal conditions. Zettl's team is currently working on the method to integrate this nanotube radio with biological systems, and this will help in understanding cell biology at the molecular level (Sur, 2008).

\subsection{Development of carbon nanotube loudspeaker}

In a recent development, researchers in China had claimed that they were able to develop a paper-thin loudspeaker that can either be attached to clothing, walls, windows or even can be inserted into the ear. These paper-thin loudspeakers were made from transparent carbon nanotube films and their work was published in the ACS journal Nano Letters (Fan et al. 2008). Professor Fan and his research group at the Tsinghua University, China developed the smallest loudspeaker of the world using CNT thin films by a simple fabrication process. At present, the loudspeakers available in the market are bulky and complex and by making these loudspeakers thinner and compact, these can be suitably used for new digital electronics devices and various other applications. Carbon Nanotubes (CNTs) are already being used for various applications including transmission of sound and music. Researchers mounted a carbon nanotube film on two electrodes and found that the system works as a loudspeaker. This CNT thin film can generate sounds by applying an audio frequency current through it. This phenomenon can be attributed to a thermoacoustic effect. The ultra small heat capacity per unit area of CNT thin films can lead to a wide frequency response range and a high sound pressure level. This CNT thin film can be a practical magnet-free loudspeaker, which has a very simple structure. The CNT thin film was pulled out from a superaligned CNT array grown on a 4 in. silicon wafer and put on two electrodes of a frame to make a loudspeaker (Figure 12a). The CNT thin film is composed of CNTs around $10 \mathrm{~nm}$ in diameter, which are sparsely parallel-aligned in the drawing direction as shown by the SEM image (Figure $12 \mathrm{~b}$ ). It is tens of nanometers thick, extremely lightweight (typical mass per unit area is $1.5 \mu \mathrm{g} / \mathrm{cm} 2)$, transparent $(78 \%$ at $550 \mathrm{~nm}$ for a single-layer CNT thin film), and conductive (typical sheet resistance is around $1 \mathrm{k} \Omega$ per square for a single-layer CNT thin film). The ac impedance of a CNT thin film is pure resistance and shows no frequency dependence within $1 \mathrm{MHz}$. Several CNT thin films can be put together to make a large area loudspeaker. As CNT can be tailored into many shapes and sizes, one can fabricate loudspeakers with special functions. Figure $12 \mathrm{~d}$ shows the cylindrical cage-like CNT thin film loudspeaker, which can emit sounds in all directions. Such a single-element loudspeaker can also be placed on a variety of rigid or flexible insulating surfaces. Two kinds of CNT thin films loudspeakers were fabricated for testing, i.e., one layer and four layer loudspeakers. In case of the CNT thin film loudspeaker, the sound generation can be understood with the aid of a thermoacoustic mechanism. The alternating current periodically heated the CNT thin films, resulting in a temperature oscillation. The 
temperature oscillation of the thin film excites the pressure oscillation in the surrounding air, resulting in the sound generation. In this process, it is the thermal expansion and contraction of the air in the vicinity of the thin film that produces sound, not the mechanical movement of the thin film itself. A direct consequence of this thermoacoustic mechanism is that the frequency of the output doubles that of the input. For an alternating current passing through, the CNT thin film will be heated during both positive and negative half-cycles, resulting in a double frequency temperature oscillation, as well as a double frequency sound pressure. Further these carbon nanotube based loudspeakers for small area devices are also suitable for buzzers or earphones. This research will lead many more applications of carbon nanotubes.

(a)

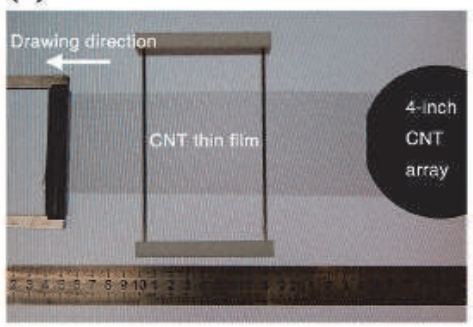

(c)

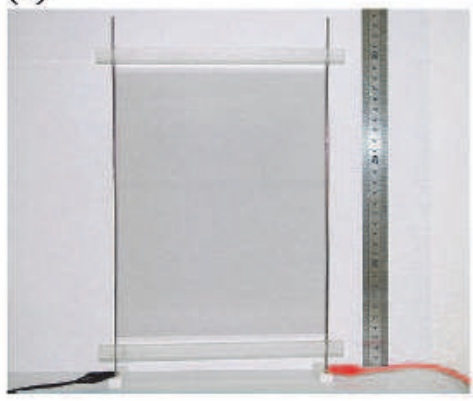

(b)

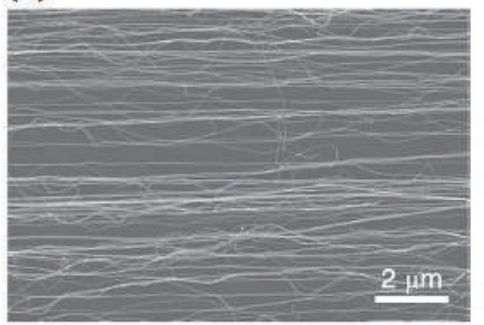

(d)

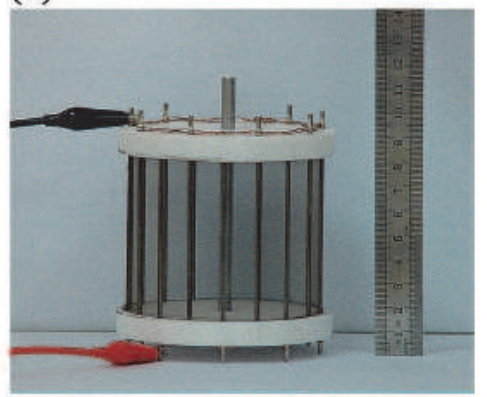

Fig. 12. Carbon nanotube thin film loudspeakers. (a) The CNT thin film was pulled out from a superaligned CNT array grown on a 4 in. silicon wafer and put on two electrodes of a frame to make a loudspeaker. (b) SEM image of the CNT thin film. (c) A4 paper size CNT thin film loudspeakers. (d) The cylindrical cage shape CNT thin film loudspeaker, which can emit sounds in all directions. (Reproduce with permission from American Chemical Society)

\section{Conclusion}

Researchers in California had developed a nanosized carbon nanotube based radio device. This single nanotube radio is about 19 orders of magnitude smaller than the Philco vacuum tube radio of the 1930s. It is expected that these carbon-nanotube based devices will replace the existing silicon (Si)-technology based devices in the near future providing an improvement in efficiency. On the other hand, researchers in China had developed a nanosized flexible, stretchable, transparent CNT thin film loudspeakers, which could open 
up new applications of carbon nanotube based electronic devices such as loudspeakers. It is expected that scientists in the near future will able to integate the nanosized radio and loudspeaker with the possibility to fabricate a fully operating radio based on carbon nanotube. For further reading, see:

http://www.physics.berkeley.edu/research/zettl/projects/nanoradio/radio.html and the article published in Current Science (Sur, 2008).

\section{Acknowledgement}

We acknowledge financial support from the project funded by the UGC, New Delhi (grant no. PSW-038/10-11-ERO). I would like to thank Dr. Frank Marken, University of Bath and Professor V. Lakshminarayanan for their valuable comments. I would like to acknowledge Zettl and co-workers, Lawrence Berkeley National Laboratory and UC Berkeley, USA for giving permission to reproduce Fig. 10 \& Fig. 11. I would like to thank the Director, Bose Institute, Kolkata for reproducing Fig. 8 \& Fig. 9. I would like to thank American Chemical Society for giving permission to reproduce Fig. 12. Finally, this chapter is dedicated to the memory of my beloved brother Kallol Kumar Sur.

\section{References}

Bose, J.C., (1897) On the determination of the wavelength of electric radiation by a diffraction grating. Proc. R. Soc. London, Vol. 60, (December 1897), pp. 167-178, ISSN 1471-2946.

Ebbesen, T.W., \& Ajayan, P. M., (1992) Large-Scale Synthesis of Carbon nanotubes. Nature, Vol. 358, ( 16 July 1992), pp. 220-222, ISSN 0028-0836.

Guo, T., Nikolaev, P.N., Thess A., Colbert, D.T., \& Smalley, R.E., (1995) Catalytic Growth of Single-walled nanotube by laser vaporization. Chemical Physics Letter, Vol. 243, (8 September 1995), pp. 49-54, ISSN 0009-2614.

Iijima, S., (1991) Helical microtubules of graphitic Carbon. Nature, Vol. 354, (7 November 1991), pp. 56-58. ISSN 0028-0836.

Jensen, K., Weldon, J., Garcia, H. \& Zettl, A., (2007) Nanotube Radio. Nano Lett., Vol. 7, No. 12, (December 2007), pp. 3508-3511, ISSN 1530-6984.

Kroto, H. W., Heath, J. R., O'Brien, S. C., Curl, R. F., \& Smalley, R. E. (1985). C60: Buckminsterfullerene. Nature, Vol. 318, (14 November 1985), pp.162-163, ISSN 0028-0836.

Rutherglen, C., \& Burke, P., (2007) Carbon Nanotube Radio. Nano Lett., Vol. 7, No. 11, (November 2007), pp. 3296-3299, ISSN 0028-0836.

Sur, U.K. (2008) World's first single carbon nanotube radio. Current Science, Vol. 94, No. 2, (25 January 2008), pp. 166-167, ISSN 0011-3891.

Xiao, L., Chen, Z., Feng, C., Liu, L., Bai, Z-Q., Wang, Y., Qian, L., Zhang, Y., Li, Q, Jiang, K., \& Fan, S. (2008) Flexible, Stretchable, Transparent Carbon Nanotube Thin Film Loudspeakers. Nano Lett., Vol. 8, No. 12, (December 2008), pp. 4539-4545, ISSN 0028-0836. 


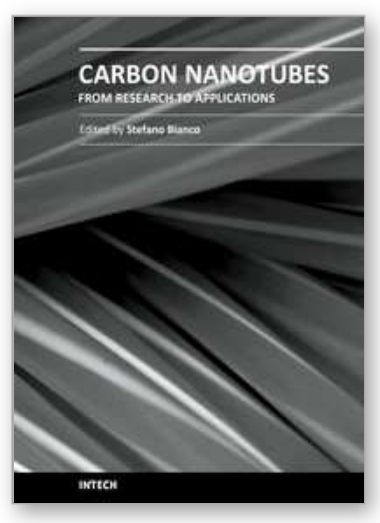

\author{
Carbon Nanotubes - From Research to Applications \\ Edited by Dr. Stefano Bianco
}

ISBN 978-953-307-500-6

Hard cover, 358 pages

Publisher InTech

Published online 20, July, 2011

Published in print edition July, 2011

Since their discovery in 1991, carbon nanotubes have been considered as one of the most promising materials for a wide range of applications, in virtue of their outstanding properties. During the last two decades, both single-walled and multi-walled CNTs probably represented the hottest research topic concerning materials science, equally from a fundamental and from an applicative point of view. There is a prevailing opinion among the research community that CNTs are now ready for application in everyday world. This book provides an (obviously not exhaustive) overview on some of the amazing possible applications of CNT-based materials in the near future.

\title{
How to reference
}

In order to correctly reference this scholarly work, feel free to copy and paste the following:

Ujjal Kumar Sur (2011). Carbon Nanotube Radio, Carbon Nanotubes - From Research to Applications, Dr. Stefano Bianco (Ed.), ISBN: 978-953-307-500-6, InTech, Available from:

http://www.intechopen.com/books/carbon-nanotubes-from-research-to-applications/carbon-nanotube-radio

\section{INTECH}

open science | open minds

\section{InTech Europe}

University Campus STeP Ri

Slavka Krautzeka 83/A

51000 Rijeka, Croatia

Phone: +385 (51) 770447

Fax: +385 (51) 686166

www.intechopen.com

\section{InTech China}

Unit 405, Office Block, Hotel Equatorial Shanghai

No.65, Yan An Road (West), Shanghai, 200040, China

中国上海市延安西路65号上海国际贵都大饭店办公楼 405 单元

Phone: +86-21-62489820

Fax: +86-21-62489821 
(C) 2011 The Author(s). Licensee IntechOpen. This chapter is distributed under the terms of the Creative Commons Attribution-NonCommercialShareAlike-3.0 License, which permits use, distribution and reproduction for non-commercial purposes, provided the original is properly cited and derivative works building on this content are distributed under the same license. 